\title{
Successfully conducting an objective structured clinical examination with real patients during the COVID-19 pandemic
}

\author{
CH Lee \#, MB, BS, Pauline Y Ng \#, MB, BS, Shirley YY Pang \#, MD, David CL Lam, MD, CS Lau *, MD
}

This article was published on 2 Feb 2021 at www.hkmj.org.
Department of Medicine, The University of Hong Kong, Hong Kong

\# These authors contributed equally to this commentary.

* Corresponding author: cslau@hku.hk

Hong Kong Med J 2021;27:61-3

https://doi.org/10.12809/hkmj208839

\section{Introduction}

For decades, the objective structured clinical examination (OSCE) has been regarded as a valid and reliable method of assessing clinical competency in medical education. The choice of patient representation in an OSCE depends on context and availability. ${ }^{1}$ The coronavirus disease 2019 (COVID-19) pandemic presents an unprecedented challenge to both medical education and examinations worldwide, especially high-stakes qualification examinations such as the final-year medical student OSCE. Whereas some medical schools elected to cancel all clinical examinations and grant provisional registration to graduates, others have resorted to the use of manikins, video recordings, or simulated patients for assessment of clinical skills. ${ }^{2}$ Hong Kong was one of the earliest regions in the world to be affected by COVID-19. ${ }^{3}$ Owing to uncertainty about the scale of the local outbreak, the Department of Medicine at The University of Hong Kong decided to uphold the format of its final-year OSCE by involving assessment of real patients in all clinical stations. We hereby report the organisation details of our OSCE conducted during the COVID-19 pandemic, with a focus on adaptations that were necessary to address concerns about infection control, whilst maintaining the overall validity of the examination.

\section{Objective structured clinical examination design and structure}

Our OSCE for the graduating class of 2020 was conducted from 4 to 8 May 2020. A total of 204 candidates, divided into 26 cohorts, were examined. As in previous years, each candidate was examined in a circuit of eight stations within 80 minutes, consisting of five clinical stations with real patients, two data interpretation stations, and one on ethics and communication with a trained actor. Candidates were examined at each clinical station by a pair of physician examiners, who separately graded their performance based on a standardised marking scheme, with emphasis on the approach to patients, core examination skills, and ability to detect and interpret physical signs.
In the past, our medical OSCE had always been conducted in the ward areas of Queen Mary Hospital, Hong Kong. However, following our first confirmed COVID-19 patient on 22 January 2020 and activation of the Emergency Response Level in public hospitals 3 days later mandating that all teaching activities conducted within hospital compounds be suspended, ${ }^{4}$ our OSCE had to be relocated to a non-clinical area on campus. Seminar rooms had to be temporarily converted to patient cubicles using portable ward screens, and a total of five seminar rooms were employed to construct the OSCE circuit (Fig).

Altogether, 52 volunteer patients from the community were recruited, with an average of 24 patients participating on each day of the examination due to multiple attendances. Since most clinical staff were deployed to combat COVID-19, a crew of 70 research assistants, laboratory technicians, post-doctoral fellows and administrative staff, who mostly had no prior experience with the OSCE, was enlisted as administrators and helpers.

\section{Infection control measures}

Our OSCE was also conducted when the Prohibition on Group Gathering Regulation, which limited group gatherings of more than four people in public places, was in force. Our Faculty successfully acquired exemption from this Regulation for our OSCE. Since close physical contact was inevitable during clinical examination and in order to safeguard all participants, several faculty-wide stringent precautionary infection control measures were implemented.

Firstly, all participants, including candidates, examiners, patients and helpers, submitted a deep throat saliva specimen for severe acute respiratory syndrome coronavirus 2 reverse-transcription polymerase chain reaction test at least 2 days prior to their OSCE participation. Secondly, all participants were required to submit a health declaration form 1 day prior to each day of examination. Those who declared any suggestive symptom in the past 7 days, ${ }^{5}$ 


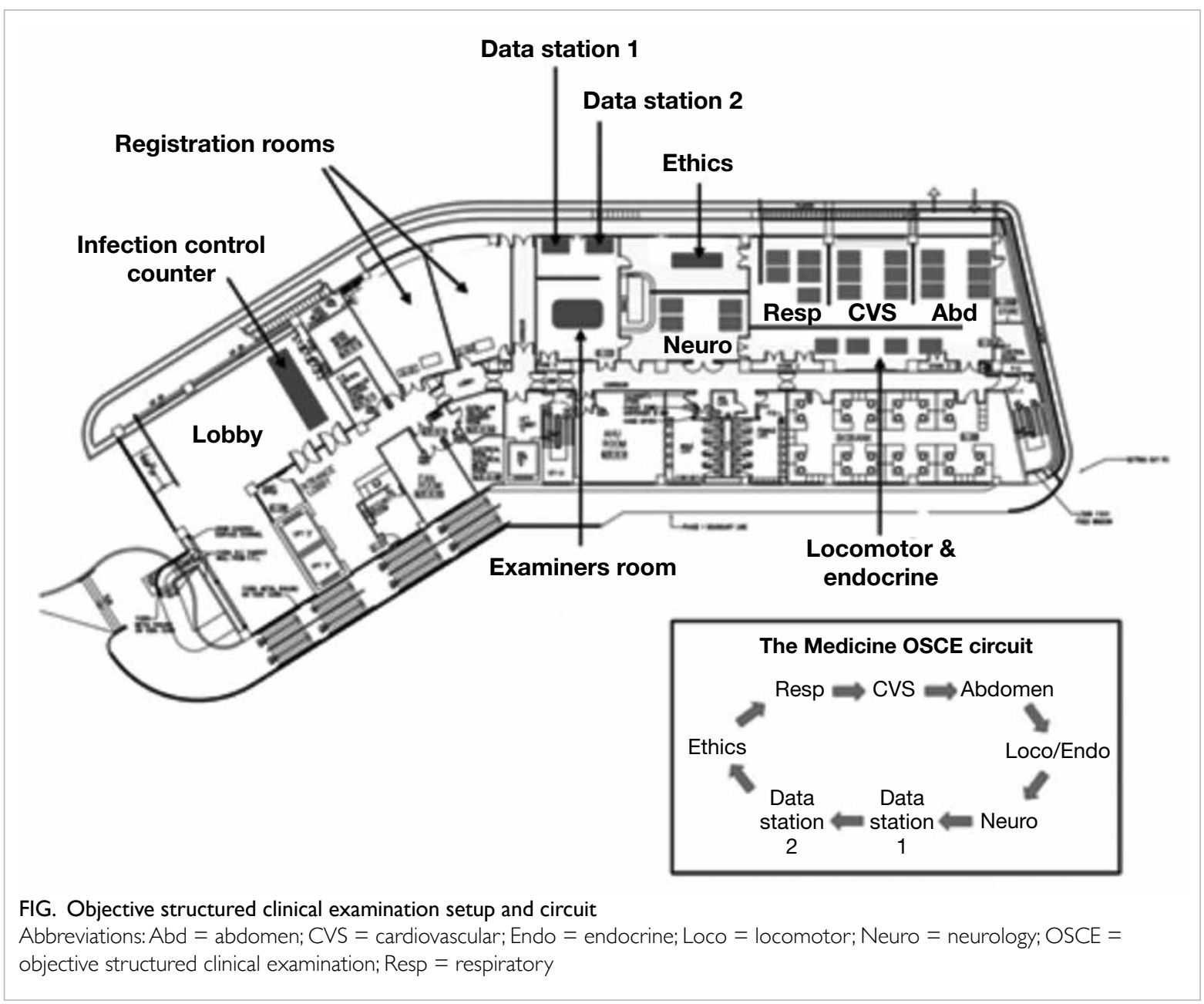

history of travel or contact with confirmed or suspected COVID-19 patients in the past 14 days, were flagged for further considerations before being allowed to participate in the examination on the following day. Thirdly, participants who persistently had a body temperature of $\geq 37.5^{\circ} \mathrm{C}$ measured on arrival at the examination venue were subjected to clinical assessment by the on-duty infection control supervisor to determine if they would be permitted to participate. Protocols were in place for clinical assessment of candidates for these purposes.

At all times during the OSCE, participants were required to wear surgical masks, and examiners and candidates had been instructed not to unmask a patient at any time. Demonstration of cough manoeuvre was also not mandatory. To minimise close contact, fundoscopic examination was replaced by interpretation of retinal photos in the data stations. Participants were required to perform strict hand hygiene using alcohol-based hand rub and disinfect their clinical instruments before and after examining each patient. Social distancing was observed in all waiting areas, with a spacing of 2-metre radius between seats. All bedsheets, pillows, and pyjamas of patients were changed daily, and the examination hall was disinfected twice daily. Lunch and drinks were all individually packaged and consumed within each individual's assigned station.

At the conclusion of our OSCE, only one candidate did not attend the examination because of persistent fatigue and will be allowed to sit in the Supplementary Examination. No candidates were denied entry on the day of examination. A total of 53 clinical teachers assessed the candidates at the clinical examination stations. All volunteering patients had negative COVID-19 tests and there were at least three patients in each clinical station daily. No major equipment failure occurred during OSCE, except for a slight delay in the electronic scoring system on the first day of the examination, which were immediately resolved by our on-site computer technicians. The candidates' pass and fail rates were similar to previous OSCEs. Importantly, no participants have contracted COVID-19 through participation in the examination. 


\section{Difficulties and limitations}

The implementation of the above measures was not without difficulties, and there remain limitations worth highlighting. Although an out-of-hospital venue was reassuring to patients who wished to avoid hospital attendance during the pandemic, this limited the diversity of patients, especially in terms of their disease severity, represented in our OSCE. Some patients who had participated in OSCEs in past years declined to help this time for fear of contracting the virus despite the out-of-hospital venue. Nonetheless, since the selection of patients was based on a list of core and optional medical competencies that students are expected to acquire during various clerkship teachings in our Department, the overall disease spectrum represented was still comparable with OSCEs in previous years.

Moreover, with compulsory quarantine imposed on all inbound travellers entering Hong Kong, special arrangements had to be made with our external examiner, who was based in the United Kingdom. A list of the patients recruited for OSCE was sent to the external examiner to evaluate diversity and appropriateness of cases, and the distinction viva was conducted over video-teleconferencing with his presence.

The COVID-19 pandemic has affected all strata of the healthcare system, and medical schools are no exception. Organising an in-person realpatient OSCE was an immense, but not impossible challenge. We demonstrated that strict infection control measures and detailed planning, albeit with a high administrative cost, could provide reassurance to all OSCE participants, facilitate the smooth running of the examination, and uphold the standard we expect from our medical graduates. Lastly, we should emphasise that good patient rapport fostered by physicians during routine clinical care is unarguably a major key to the successful conduction of our OSCE where our patients have demonstrated tremendous support and trust in the measures we have taken. While it remains uncertain when this pandemic would come to an end, or if social distancing practices have to be kept indefinitely, our successful experience may provide some guidance and encouragement to those who are pressing on to deliver safe and valid qualifying examinations during one of the most difficult times in this century.

\section{Author contributions}

Concept or design: CH Lee, CS Lau, PY Ng, SYY Pang. Drafting of the manuscript: CH Lee, PY Ng, SYY Pang. Critical revision of the manuscript for important intellectual content: DCL Lam, CS Lau.

All authors had full access to the data, contributed to the study, approved the final version for publication, and take responsibility for its accuracy and integrity.

\section{Conflicts of interest}

CS Lau is Immediate Past President of the Hong Kong Academy of Medicine. Other co-authors report no conflicts of interest.

\section{Acknowledgement}

We thank the Li Ka Shing Faculty of Medicine for its enduring support. We are grateful for the efforts and contributions from all participants, especially our patients, who have made this objective structured clinical examination possible and successful.

\section{References}

1. Collins JP, Harden RM. AMEE Medical Education Guide No. 13: real patients, simulated patients and simulators in clinical examinations. Med Teach 1998;20:508-21.

2. Boursicot K, Kemp S, Ong TH, et al. Conducting a highstakes OSCE in a COVID-19 environment. MedEdPublish 2020;9:54.

3. Centre for Health Protection, Department of Health, Hong Kong SAR Government. Latest situation of cases of COVID-19. Available from: https://www.chp.gov.hk/ files/pdf/local_situation_covid19_en.pdf. Accessed 18 May 2020.

4. Hospital Authority, Hong Kong SAR Government. Hospital Authority activates Emergency Response Level. Available from: https://www.ha.org.hk/haho/ho/pad/200125Eng6. pdf. Accessed 25 Jan 2020.

5. Huang C, Wang Y, Li X, et al. Clinical features of patients infected with 2019 novel coronavirus in Wuhan, China. Lancet 2020;395:497-506. 